\title{
Torakolomber omurga kırıklarında güncel sınıflandırmalar
}

\author{
Current classifications of thoracolumbar spine fractures
}

\author{
Serkan Erkan
}

Celal Bayar Üniversitesi Tıp Fakültesi Ortopedi ve Travmatoloji Anabilim Dalı, Manisa

\begin{abstract}
Omurganın travmatik yaralanma sıklığının, tüm kırıklar göz önüne alındığında \%4-23 arasında olduğu belirtilmektedir. Fakat, hastalar üzerindeki sosyal, fonksiyonel ve ekonomik etkileri diğer yaralanmalara göre daha belirgin olmaktadır; çünkü bu yaralanma uzun dönemde ciddi sakatlık oluşturur. Omurga kırıklarının tedavi planlamasında kırık sınıflandırmasının önemi büyüktür. İdeal bir sınıflandırma göreceli olarak basit, tekrarlanabilir, prognozu belirleyebilir ve tedaviyi yönlendirici olmalıdır. Bu özellikleri sağlayan sınıflandırmalar cerrahlar arası akademik ve pratik iletişimi kolaylaştırabilir. Geçmişteki sınıflandırma sistemleri iki veya üç kolon stabilite, yaralanma mekanizması veya özgül kemiksel yaralanma tipine dayanmaktadır. Daha yakın tarihteki sınıflandırmalar, yaralanmanın morfolojisi, posterior ligamentöz kompleks bütünlügü ve hastanın nörolojik durumunu da değerlendirmektedir. Kuzey Amerika'da omurga cerrahları daha çok Torakolomber Yaralanma Sınıflandırma Skorlaması (TLICS) kullanırken, Avrupa'da daha çok Magerl Sınıflandırması kullanılmaktadır. Güncel olarak, AOSpine Torakolomber Yaralanma Sınıflandırma Skorlaması'nda aynı anda spinal kolonun morfolojik yapısı, yetmezlik modelleri, nörolojik durum ve tedavi niteleyicileri gibi klinik özellikler dikkate alınmaktadır. Gözlemciler arası ve gözlemciler içi güvenilirliği birçok çalışmada ispatlanan AOSpine Torakolomber Yaralanma Sınıflandırma Skorlaması cerrahlar arası iletişim, tedavi planlaması ve akademik amaçlar için kullanılabilir.
\end{abstract}

Anahtar sözcükler: torakolomber omurga; omurga travması; sınıflandırma; omurga kırığı; burst kırı̆̆ı
The incidence of traumatic injuries of the spinal column compared to all fractures is reported to be $4-23 \%$. However, their influence on patients' social, functional, and financial situation is more significant than other injuries, as they may contribute to disability with long-term consequences. Fracture classification has great impact on the management of vertebra fractures. The ideal classification should be relatively simple to use, reproducible, define prognosis, and be guide for therapeutic decision making. Classification systems that meet these criteria facilitate academic and practical communication between surgeons. Historical classification systems depend on 2 or 3 column stability, mechanism of injury or specific osseous injury type. Recent classifications evaluate morphology of the injury, the integrity of the posterior ligamentous complex and neurological status of the patient. In North America, many spine surgeons use the Thoracolumbar Injury Classification System; in contrast, many European surgeons commonly use the Magerl system. Currently, AOSpine Thoracolumbar Injury classifications simultaneously consider the morphological description of spinal column injuries, all major modes of failure and clinical features such as neurological status and treatment modifiers. Several agreement studies demonstrated substantial inter- and intra-observer reliability for AOSpine Thoracolumbar Injury classification that can be used for communication between surgeons, planning treatment, and academic purposes.

Key words: thoracolumbar spine; spine trauma; classification; vertebra fracture; burst fracture

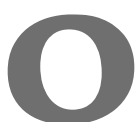

murganın travmatik yaralanma sıklığının, tüm kırıklar göz önüne alındığında \%4-23 arasında olduğu belirtilmektedir. ${ }^{[1]}$ Fakat hastalar üzerindeki sosyal, fonksiyonel ve ekonomik etkileri diğer yaralanmalara göre daha belirgin olmaktadır; çünkü bu yaralanma uzun dönemde ciddi sakatlık oluşturur. ${ }^{[2]}$ Omurga kırıklarının tedavi planlamasında kırık sınıflandırmasının önemi büyüktür. İdeal bir sınıflandırma göreceli olarak basit, tanısal olarak tekrarlanabilir, prognozu belirleyebilir ve tedaviyi yönlendirici olmalıdır. Bu özellikleri sağlayan sınıflandırmalar, cerrahlar arası akademik ve pratik iletişimi kolaylaştırabilir. ${ }^{[2]}$

Geçmişte, hem mekanik hem de yaralanmanın radyografik morfolojisine bağlı sınıflandırmalar

- İletişim adresi: Doç. Dr. Serkan Erkan, 116/8 sok. No:2 Vatansever sitesi C Blok, D:6 Bornova, İzmir Tel: 0532 - 5514535 e-posta: serkanerkan73@yahoo.com

- Geliș tarihi: 1 Kasım $2018 \quad$ Kabul tarihi: 1 Kasım 2018 
yapılmıştır. ${ }^{[3]}$ Her ne kadar çok sayıda sınıflandırma yapılmış olsa da, güncel olarak genel kabul görmüş bir sınıflandırma yoktur. Bunun nedeni, sınıflandırmalardaki güvenilirlik, doğruluk ve klinik uyumluluk eksikliğidir. [4] Kuzey Amerika'da omurga cerrahları daha çok Torakolomber Yaralanma Sınıflandırma Skorlaması (TLICS $)^{[5]}$ kullanırken Avrupa'da Magerl Sınıflandırması ${ }^{[6]}$ daha çok kullanılmaktadır. Daha güncel olarak ise, AOSpine Torakolomber Yaralanma Sınıflandırma Skorlaması (TLAOSIS) yayımlanmış ve geçerliliği onaylanmıştır. ${ }^{[7-10]}$ Fakat, bu güncel sınıflandırmaların küresel ölçekte kabul edilebilirliği henüz belirli değildir.

\section{TARIHSEL SINIFLANDIRMALAR}

1938 yılında torakolomber kırıkların sınıflandırması Watson-Jones tarafından yapılmıştır. Basit kama, parçalı ve kırıklı-çıkık şeklinde üç farklı kırık tipi tanımlanmış ve bunlar için farklı tedavi tipleri önerilmiştir. ${ }^{[11]}$ 1948 yılında Chance kırı̆̆ı tanımlanmıştır. Bu kırık tipi fleksiyon kuvetlerine bağlı vertebrada kamalaşma ve sonrasında posterior elemanların yaralanmasıdır. ${ }^{[12]}$ Diğer kırık tipi burst (patlama) kırığıdır. 1970 yılında Holdsworth tarafından arka duvarın hasarlandığı kompresyon kırı̆̆ı olarak tanımlanmıştır. ${ }^{[13]}$ Aynı yayında Holdsworth ilk mekanik sınıflamayı önermiş̧tir. Kırıkları temel olarak altı temel tipe ayırmıştır: basit kama, çıkık, rotasyonel kırıklı çıkık, ekstansiyon, burst ve makaslama yaralanması. Bu yayının en önemli ve tartışmalı bulgusu ise bütün bu kırık tiplerinde posterior ligamentöz kompleksin (PLK) hasarlanmamış ve stabil olmasıdır. Bu sınıflama her ne kadar temel tedavi prensiplerini verse de bağımsız olarak geçerliliği kanıtlanmamıştır. ${ }^{[14,15]}$

\section{KOLON SINIFLANDIRMALARI}

1968 yılında Kelly ve Whiteside ${ }^{[16]}$, omurgayı iki kolona bölerek ikinci en büyük sınıflandırmayı yapmışlardır. Anterior kolon içeriği olarak tüm vertebral cisim ve intervertebral disk, posterior kolon içeriği ise nöral ark ve PLK olarak belirlenmiştir. Yaralanma tek bir kolonu tutuyorsa stabil, her iki kolonu tutuyorsa instabil olarak kabul edilmiştir. ${ }^{[16]}$ Bu sınıflandırmanın da hiçbir zaman bağımsız olarak geçerliliği kanıtlanamamıştır. 1983 yılında Denis, her bir spinal segmenti üç kolona bölmüştür: anterior kolon, anterior longitudinal bağ ile vertebral cismin anterior 2/3'ü; orta kolon, vertebral cismin posterior $1 / 3$ 'ü ile posterior longitudinal bağ; posterior kolon ise, posterior longitudinal bağın posteriorundaki tüm anatomik yapıları içerir. ${ }^{[17]} \mathrm{Bu}$ sınıflandırma, kırıkları dört majör tipe ayırmıştır: çökme kırıkları, burst kırıkları, emniyet kemeri tipi yaralanmalar ve kırıklı çıkıklar. Daha sonra her bir kırık tipini toplam 16 alt tipe ayırmıştır (Tablo $1)$. Denis tedavinin orta kolon bütünlüğüne göre belirlenmesini önermiştir. İzole anterior ve posterior kolon yaralanmaları stabil, ama bunlara orta kolon yaralanması eşlik ederse instabildir. ${ }^{[17]}$ Üç kolon stabilite teorisi küresel olarak kabul görmüştür ve hala günümüzde bir çok omurga cerrahı tarafından özellikle nörolojik hasarı olmayan torakolomber burst kırıklarında cerrahi tedavi kararında kullanılmaktadır. ${ }^{[10]}$ Buna karşın, çalışmacılar arası güvenirlilik düşük ve orta düzeyde bulunmuştur. ${ }^{[18]}$ Orta kolonun spinal stabilitedeki önemi, halen literatürde tartışılmaktadır.

McAfee ve ark. Denis'in çalışmasına atıfta bulunarak, hem morfolojik hem de mekanik bir sınıflandırma yayımlamışlardır. ${ }^{[19]}$ Kırıklar tipine göre altıya ayrılmıştır: çökme, stabil burst, instabil burst, fleksiyon distraksiyon, Chance ve translasyonel yaralanma. Denis sınıflandırmasına benzer şekilde üç kolon tanımlanmış ve orta kolonu içeren tüm kırıklar instabil kabul edilmiştir. McAfee ve ark., orta kolon yetmezliğinin uygun tedavinin belirlenmesinde önemli olduğunu vurgulamışlardır. ${ }^{[19]}$

Tablo 1. Denis sınıflaması

\begin{tabular}{cl}
\hline Kompresyon (anterior veya lateral) \\
\hline Tip A & Anterior kolon koronal split \\
Tip B & Anterior kolon superior uç plak kırığı \\
Tip C & Anterior kolon inferior uç plak kırı̆ıı \\
Tip D & Uç plakların sağlam olduğu anterior korteks kırığı \\
\hline Burst & \\
\hline Tip A & Her iki uç plak ve arka duvarı içeren kırık \\
Tip B & Superior uç plak ve arka duvarı içeren kırık \\
Tip C & Inferior uç plak ve arka duvarı içeren kırık \\
Tip D & Rotasyonun eşlik ettiği burst kırı̆ıı \\
Tip E & Her iki uç plak ve arka duvarı içeren lateral burst \\
\hline kırığı \\
\hline Emniyet kemeri tipi \\
\hline Tip A & Tek seviyeli osseöz yaralanma \\
Tip B & Tek seviyeli ligamentöz yaralanma \\
Tip C & Orta kolonun osseöz tutulumun olduğu iki \\
Tip D & seviyeli yaralanma \\
& Orta kolonun ligamentöz tutulumun olduğu iki \\
seviyeli yaralanma
\end{tabular}


1984 yılında Ferguson ve Allen sınıflandırması yayımlanmıştır. ${ }^{[20]}$ Bu sınıflama mekanik bir sınıflama olup Denis'in üç kolon biyomekanik stabilite tanımı ile çelişmez. Bu nedenle, tedavi prensipleri açısından literatüre katkısı azdır. Bu sınıflandırma yedi ana yaralanma tipi ile beş alt yaralanma tipini içerir (Tablo 2) ve bağımsız olarak geçerliliği de kanıtlanamamıştır.

1994 yılında Magerl ve ark. yeni bir sınıflandırma tanımlamışlardır. ${ }^{[6]}$ Magerl sınıflandırmasına göre kırıklar üç tipe ayrılmıştır: Kompresyon/Tip A kırıklar, Distraksiyon/Tip B kırıklar, Rotasyonel/Tip C kırıklar. Bunlar da morfolojik tiplerine göre 53 alt gruba ayrılmaktadır. Bu sınıflandırma, her ne kadar Tip A'dan C'ye ilerledikçe yaralanma şiddeti artış gösteriyor olsa da, Denis'in üç kolon biyomekanik stabilite teorisine dayandırılmıştır. Bu nedenle, tedavi algoritmasına katkısı sınırlı kalmıştır. Sınıflandırmanın karmaşıklığı sonucu, çalışmacılar arası güvenirlilik oranları zayıf ve yetersiz bulunmuştur. ${ }^{[18,21]}$ Buna bağlı olarak, klinik geçerliliği kanıtlanamamıştır. Bu sınıflandırmanın diğer zayıf yönü ise PLK bütünlüğünün ve hastanın nörolojik durumunun sınıflandırmaya dahil edilmemesidir. ${ }^{[6]}$

2005 yılında Vaccaro ve ark. Torakolomber Yaralanma Sınıflandırma Skorlamasını yayımlamışlardır. ${ }^{[5]}$ Yazarlar bu sınıflandırmada, daha önceden kabul edilmiş 'Tüm orta kolon kırıkları instabildir.' görüşüne karşı çıkmışlardır (Tablo 3). Bu sınıflandırma PLK'nın yapısal bütünlüğü ve hastanın nörolojik durumunu sorgulayan ilk sınıflamadır. Kırıklar önce morfolojik olarak üç gruba ayrılmaktadır: çökme tipi yaralanma, translasyonel/rotasyonel yaralanma ve distraksiyon yaralanması. İkinci aşamada PLK bütünlüğü: intakt, hasarlı ve belirsiz şeklinde

Tablo 2. Ferguson ve allen sınıflaması

\begin{tabular}{l}
\hline Vertikal kompresyon \\
\hline Belirgin retropulsiyonu olan burst kırı̆̆ı \\
Superior ve inferior uç plakta retropulsiyonu olan burst kırığı \\
\hline Kompresyon fleksiyon \\
\hline Anterior kama \\
Posterior tansiyon bandı yaralanması ile anterior kama \\
Posterior tansiyon bandı yaralanması ile burst kırığı \\
Fleksiyon distraksiyon \\
Lateral fleksiyon \\
Translasyon \\
Torsiyonel fleksiyon
\end{tabular}

değerlendirilmektedir. Son olarak da, hastanın nörolojik durumu: intakt, kök hasarı ve komplet spinal kord hasarı veya inkomplet spinal kord hasarı / kauda ekina sendromu olarak değerlendirilmektedir. ${ }^{[5]}$ Buna ek olarak, yaralanmanın sınıflandırılması haricinde her bir durum için puanlama yapılarak yaralanma şiddeti skoru belirlenmektedir. Bu durum tedavi açısından da yönlendiricidir: Toplam skor 4'ün altındaysa cerrahi dışı tedavi uygulanmakta, 4 ise tedavi seçimi hastanın durumuna bağlı olarak cerraha bırakılmakta, 4'ün üzerinde ise cerrahi tedavi önerilmektedir. Yapılan bir çalışmada, bu sınıflamanın geçerliliği \%96,2 oranında sağlanmıştır. ${ }^{[22]}$ Ayrıca, pediatrik hasta grubunda Savage ve ark. da bu sınıflandırmanın geçerliliğini kanıtlamışlardır ( 0,84 duyarlılık, 0,79 özgüllük, 0,68 pozitif öngörü değeri ve 0,90 negatif öngörü değeri). ${ }^{[23]}$

Her ne kadar literatürde bu sınıflandırmanın geçerliliği kanıtlanmış olsa da, küresel ölçekte kabul edilebilirliği yetersiz kalmaktadır; çünkü burst kırı̆̆ı olan nörolojik intakt hastalarda tedavi önerisi PLK bütünlüğüne bağlıdır. Eğer PLK intakt ise cerrahi dışı tedavi, PLK hasarlı ise cerrahi tedavi önerilmektedir. Sonuç olarak; eğer PLK'nın durumu belirsiz ise, bu sınıflandırmada tedavi önerisi yetersiz kalmaktadır. Günümüz koşullarındaki görüntüleme yöntemleriyle, bu kırık tiplerinde PLK bütünlüğü açısından görüş ayrılığı olmaktadır. ${ }^{[3]}$

Tablo 3. Torakolomber yaralanma sınıflandırma skorlaması

\begin{tabular}{ll}
\hline Yaralanma tipi & Skor \\
\hline Morfoloji & 1 \\
\hline Kompresyon kırı̆ı & 2 \\
Burst kırı̆̆ı & 3 \\
Rotasyonel ve translasyonel yaralanma & 4 \\
$\quad$ Distraksiyon yaralanması & \\
\hline Posterior ligamentöz kompleks & 0 \\
\hline İntakt & 2 \\
Belirsiz & 3 \\
$\quad$ Hasarlı & 0 \\
\hline Nörolojik durum & 2 \\
\hline İntakt & 2 \\
Kök hasarı & 3 \\
Komplet spinal kord hasarı &
\end{tabular}




\section{AOSPINE TORAKOLOMBER YARALANMA SINIFLANDIRMA SKORLAMASI (TLAOSIS)}

Daha önceki sınıflandırmaların küresel kabul edilebilirliğinin düşük olması üzerine, 2013 yılında Vaccaro ve ark., Magerl ve TLICS sınıflandırmalarını birleştirerek yeni AOSpine Torakolomber Yaralanma Sınıflandırma Skorlaması'nı yayımladılar. ${ }^{[24]}$ Bu sınıflandırma sistemi, tedavi algoritmasını belirlemek için modifiye Delphi metodunu kullanan ilk sınıflama özelliğini taşımaktadır. Bunun için yazarlar, önce başlangıç sınıflandırmasını yayımlamışlar, daha sonra tedavi algoritmasını yayımlamadan önce uzun dönem takipli birçok çalışma yapmışlardır. ${ }^{[7-9,25]}$

Morfolojik sınıflandırma, Magerl sisteminin basitleştirilmiş şeklidir ve toplamda dokuz yaralanma modelini içerir. Yaralanmalar başta üç ana gruba ayrılır:
A- Kompresyon yaralanmaları,
B- Tansiyon bandı yaralanmaları,
C- Translasyonel yaralanmalar.

Sonra, A grubu beş ve B grubu üç alt gruba ayrılmaktadır (Tablo 4). TLICS'e benzer şekilde, hastanın nörolojik durumu ve iki adet hastaya özgü niteleyiciler sınıflandırmaya dahil edilmiştir (Tablo 5). Kepler ve ark. çaIışmalarında, TLAOSIS sınıflandırması için gözlemciler arası güvenirlilik $(\kappa=0,74)$ ve üç kırık tipi için mükemmel

Tablo 4. Aospine torakolomber yaralanma sınıflandırma skorlaması

\begin{tabular}{|c|c|c|}
\hline Alt grup & Tanım & TLAOSIS \\
\hline \multicolumn{3}{|c|}{ Tip A-kompresyon kırıkları } \\
\hline $\mathrm{A} 0$ & $\begin{array}{l}\text { Omurganın yapısal bütünlüğünü } \\
\text { bozmayan yaralanma (spinöz veya } \\
\text { transvers çıkıntı kırığı) }\end{array}$ & 0 \\
\hline A1 & $\begin{array}{l}\text { Arka duvarı etkilemeyen tek bir uç plağı } \\
\text { tutan kırık }\end{array}$ & 1 \\
\hline A2 & $\begin{array}{l}\text { Arka duvarı etkilemeyen ve her iki uç } \\
\text { plağı tutan kırık }\end{array}$ & 2 \\
\hline A3 & $\begin{array}{l}\text { Arka duvarı tutan ve tek bir uç plağı } \\
\text { tutan kırık (inkomplet burst) }\end{array}$ & 3 \\
\hline A4 & $\begin{array}{l}\text { Arka duvarı tutan ve her iki uç plağı } \\
\text { tutan kırık (komplet burst) }\end{array}$ & 5 \\
\hline \multicolumn{3}{|c|}{ Tip B-tansiyon bandı yaralanmaları } \\
\hline B1 & $\begin{array}{l}\text { Komplet kemiksel tansiyon bandı } \\
\text { yaralanması (kemiksel chance kırığı) }\end{array}$ & 5 \\
\hline B2 & Posterior tansiyon bandı yaralanması & 6 \\
\hline B3 & Anterior tansiyon bandı yaralanması & 7 \\
\hline \multicolumn{3}{|c|}{ Tip C-translasyonel yaralanmalar } \\
\hline$C$ & $\begin{array}{l}\text { Vertebral cismin translasyonuna neden } \\
\text { olan yaralanma }\end{array}$ & 8 \\
\hline
\end{tabular}

gözlemci içi güvenirlilik $(\kappa=0,81)$ oranları bularak, bu sınıflamanın geçerliliğini kanıtlamışlardır. ${ }^{[7]} \mathrm{Bu}$ çalışmaların sonuçlarının geçerliliği, Urrutia ve Azimi'nin çalışmalarında da kanıtlanmıştır. ${ }^{26,27]}$ TLICS sınıflandırmasına benzer şekilde, TLAOSIS sınıflandırmasında da her bir değişken için puan verilerek toplam bir TLAOSIS skoru elde edilir. Sınıflandırmaya karşı bölgesel olarak oluşabilecek karşı çıkışları engellemek için dünyanın tüm bölgelerinden 500'ün üzerinde cerrah çalışmaya dahil edilerek tedavi algoritması oluşturulmuştur. Yaralanma tipi için cerrahların \%30'unun altında cerrahi önerisi geldiyse cerrahi dışı tedavi, \%70'in üstünde cerrahi önerisi varsa cerrahi tedavi önerilir. ${ }^{[3]}$ Buna göre, toplam TLAOSIS skoru 4'ün altındaki olgularda cerrahi dışı tedavi, 5 'in üzerindeki olgularda erken cerrahi tedavi önerilmektedir. Toplam TLAOSIS skoru 4 veya 5 olanlarda ise hasta ve cerrahın değişkenliğine göre karar verilmekte, çünkü hem cerrahi dışı hem de cerrahi tedavi uygun olmaktadır. ${ }^{[3]}$

\section{ÇIKARIM}

Günümüzde, torakolomber travmaların tedavisinde bölgelere göre cerrahlar arasında değişkenlikler vardır. Bunun bir nedeni de, küresel ölçekte kabul edilebilir bir sınıflandırma sisteminin yokluğudur. Geçmişteki sınıflandırma sistemleri, iki veya üç kolon stabilite, yaralanma mekanizması veya özgül kemiksel yaralanma tipine dayanmaktaydı. Daha yakın tarihteki sınıflandırmalar, yaralanmanın morfolojisini, PLK bütünlüğünü ve hastanın nörolojik durumunu da değerlendirmektedir. En son olarak yapılan TLAOSIS sınıflandırmasında ise; spinal kolonun morfolojik yapısı, yetmezlik modelleri,

Tablo 5. Aospine torakolomber yaralanma sınıflandırma skorlaması durum ve niteleyicileri

\begin{tabular}{clc}
\hline Alt grup & Tanım & TLAOSIS \\
\hline & Nörolojik durum & \\
\hline N0 & Nörolojik yaralanma yok & 0 \\
N1 & İyileşmiş geçici nörolojik yaralanma & 1 \\
N2 & Sinir kökü hasarı & 2 \\
N3 & İnkomplet spinal kord veya kauda ekina & 4 \\
& sendromu & \\
N4 & Komplet spinal kord yaralanması & 4 \\
Nx & $\begin{array}{l}\text { Güvenilir nörolojik muayene mümkün } \\
\text { değil }\end{array}$ & 3 \\
\hline \multirow{2}{*}{ M1 } & Hastaya özgü niteleyiciler & 1 \\
M2 & PLK bütünlüğü net değil & \\
& $\begin{array}{l}\text { Hastaya özgü tedaviyi etkileyecek } \\
\text { (örn; ankilozan spondilit) }\end{array}$ & 0 \\
&
\end{tabular}


nörolojik durum ve tedavi niteleyicileri gibi klinik özellikler dikkate alınmaktadır. Gözlemciler arası ve gözlemciler içi güvenilirliği birçok çalışmada ispatlanan TLAOSIS sınıflandırması, cerrahlar arası iletişim, tedavi planlaması ve akademik amaçlar için kullanılabilir.

\section{KAYNAKLAR}

1. Oliver $M$, Inaba $K$, Tang $A$, Branco BC, Barmparas $G$, Schnüriger B, Lustenberger T, Demetriades D. The changing epidemiology of spinal trauma: a 13-year review from a Level I trauma centre. Injury 2012;43(8):1296-300. Crossref

2. Oner C, Rajasekaran S, Chapman JR, Fehlings MG, Vaccaro AR, Schroeder GD, Sadigi S, Harrop J. Spine Trauma What are the current controversies? J Orthop Trauma 2017;31(Suppl 4):S1-6. Crossref

3. Schroeder GD, Harrop JS, Vaccaro AR. Thoracolumbar Trauma Classification. Neurosurg Clin N Am 2017;28(1):239. Crossref

4. Schnake KJ, Schroeder GD, Vaccaro AR, Oner C. AOSpine Classification Systems (Subaxial, Thoracolumbar). J Orthop Trauma 2017;31(Suppl 4):S14-23. Crossref

5. Vaccaro AR, Lehman RA Jr, Hurlbert RJ, Anderson PA, Harris M, Hedlund R, Harrop J, Dvorak M, Wood K, Fehlings MG, Fisher C, Zeiller SC, Anderson DG, Bono CM, Stock GH, Brown AK, Kuklo T, Oner FC. A new classification of thoracolumbar injuries: the importance of injury morphology, the integrity of the posterior ligamentous complex, and neurologic status. Spine (Phila Pa 1976) 2005;30(20):2325-33. Crossref

6. Magerl F, Aebi M, Gertzbein SD, Harms J, Nazarian S. A comprehensive classification of thoracic and lumbar injuries. Eur Spine J 1994;3(4):184-201. Crossref

7. Kepler C, Vaccaro A, Koerner J, Dvorak MF, Kandziora F, Rajasekaran S, Aarabi B, Vialle LR, Fehlings MG, Schroeder GD, Reinhold M, Schnake KJ, Bellabarba C, Öner FC. Reliability analysis of the AOSpine thoracolumbar spine injury classification system by a worldwide group of native spinal surgeons. Eur Spine J 2016;25(4):1082-6. Crossref

8. Schroeder GD, Kepler CK, Koerner JD, Chapman JR, Bellabarba C, Oner FC, Reinhold M, Dvorak MF, Aarabi B, Vialle L, Fehlings MG, Rajasekaran S, Kandziora F, Schnake $\mathrm{KJ}$, Vaccaro AR. Is there a regional difference in morphology interpretation of A3 and A4 fractures among different cultures? J Neurosurg Spine 2016;24(2):332-9. Crossref

9. Schroeder GD, Vaccaro AR, Kepler CK, Koerner JD, Oner FC, Dvorak MF, Vialle LR, Aarabi B, Bellabarba C, Fehlings MG, Schnake KJ, Kandziora F. Establishing the injury severity of thoracolumbar trauma: confirmation of the hierarchical structure of the AOSpine thoracolumbar spine injury classification system. Spine (Phila Pa 1976) 2015;40(8):E498-503. Crossref

10. Vaccaro AR, Schroeder GD, Kepler CK, Cumhur Oner F, Vialle LR, Kandziora F, Koerner JD, Kurd MF, Reinhold M, Schnake KJ, Chapman J, Aarabi B, Fehlings MG, Dvorak MF. The surgical algorithm for the AOSpine thoracolumbar spine injury classification system. Eur Spine J 2016;25(4):1087-94. Crossref

11. Watson-Jones $R$. The results of postural reduction of fractures of the spine. J Bone Joint Surg Am 1938;20(3):567-86.

12. Chance GQ. Note on a type of flexion fracture of the spine. $\mathrm{Br}$ J Radiol 1948;21(249):452-3. Crossref

13. Holdsworth F. Fractures, dislocations, and fracturedislocations of the spine. J Bone Joint Surg Am 1970;52(8):1534-51. Crossref
14. Mirza SK, Mirza AJ, Chapman JR, Anderson PA. Classifications of thoracic and lumbar fractures: rationale and supporting data. J Am Acad Orthop Surg 2002;10(5):364-77. Crossref

15. Patel AA, Vaccaro AR. Thoracolumbar spine trauma classification. J Am Acad Orthop Surg 2010;18(2):63-71. Crossref

16. Kelly RP, Whitesides TEJr. Treatment of lumbodorsal fracturedislocations. Ann Surg 1968;167(5):705-17. Crossref

17. Denis F. The three column spine and its significance in the classification of acute thoracolumbar spinal injuries. Spine (Phila Pa 1976) 1983;8(8):817-31. Crossref

18. Oner FC, Ramos LM, Simmermacher RK, Kingma P, Diekerhof C, Dhert W, Verbout A. Classification of thoracic and lumbar spine fractures: problems of reproducibility. A study of 53 patients using CT and MRI. Eur Spine J 2002;11(3):235-45. Crossref

19. McAfee PC, Yuan HA, Fredrickson BE, Lubicky JP. The value of computed tomography in thoracolumbar fractures. An analysis of one hundred consecutive cases and a new classification. J Bone Joint Surg Am 1983;65(4):461-73. Crossref

20. Ferguson RL, Allen BL Jr. A mechanistic classification of thoracolumbar spine fractures. Clin Orthop Relat Res 1984;(189):77-88. Crossref

21. Aebi M. Classification of thoracolumbar fractures and dislocations. Eur Spine J 2010;19(Suppl 1):S2-7. Crossref

22. Vaccaro AR, Baron EM, Sanfilippo J, Jacoby S, Steuve J, Grossman E, DiPaola M, Ranier P, Austin L, Ropiak R, Ciminello M, Okafor C, Eichenbaum M, Rapuri V, Smith E, Orozco F, Ugolini P, Fletcher M, Minnich J, Goldberg G, Wilsey J, Lee JY, Lim MR, Burns A, Marino R, DiPaola C, Zeiller L, Zeiler SC, Harrop J, Anderson DG, Albert TJ, Hilibrand AS. Reliability of a novel classification system for thoracolumbar injuries: the thoracolumbar injury severity score. Spine (Phila Pa 1976) 2006;31(11 Suppl):S62-9. Crossref

23. Savage JW, Moore TA, Arnold PM, Thakur N, Hsu WK, Patel AA, McCarthy K, Schroeder GD, Vaccaro AR, Dimar JR, Anderson PA. The reliability and validity of the thoracolumbar injury classification system in pediatric spine trauma. Spine (Phila Pa 1976) 2015;40(18):E1014-8. Crossref

24. Vaccaro AR, Oner C, Kepler CK, Dvorak M, Schnake K, Bellabarba C, Reinhold M, Aarabi B, Kandziora F, Chapman $J$, Shanmuganathan R, Fehlings $M$, Vialle L. AOSpine thoracolumbar spine injury classification system: fracture description, neurological status, and key modifiers. Spine (Phila Pa 1976) 2013;38(23):2028-37. Crossref

25. Sadiqi S, Oner FC, Dvorak MF, Aarabi B, Schroeder GD, Vaccaro AR. The influence of spine surgeons' experience on the classification and intraobserver reliability of the novel AOSpine thoracolumbar spine injury classification system - an international study. Spine (Phila Pa 1976) 2015;40(23):E1250-6. Crossref

26. Urrutia J, Zamora T, Yurac R, Campos M, Palma J, Mobarec $\mathrm{S}$, Prada C. An independent interobserver reliability and intraobserver reproducibility evaluation of the new AOSpine thoracolumbar spine injury classification system. Spine (Phila Pa 1976) 2015;40(1):E54-8. Crossref

27. Azimi P, Mohammadi HR, Azhari S, Alizadeh P, Montazeri A. The AOSpine thoracolumbar spine injury classification system: a reliability and agreement study. Asian J Neurosurg 2015;10(4):282-5. Crossref 